\title{
A Semester Like No Other: Use of Natural Language Processing for Novice-Led Analysis on End-of-Semester Responses on Students' Experience of Changing Learning Environments Due to COVID-19
}

\section{Dr. Sreyoshi Bhaduri, McGraw Hill}

Dr. Sreyoshi Bhaduri is an Engineering Educator and People Researcher. She currently heads Global People Research and Analytics at McGraw Hill, where she leads research leveraging employee data to generate data-driven insights for decisions impacting organizational Culture and Talent. Her research interests include assessing the impact and effectiveness of inclusion initiatives as well as employing innovative, ethical and inclusive mixed-methods research approaches using AI to uncover insights about the 21st century workforce. Sreyoshi is passionate about improving belonging among women in STEM and Engineering. She was recently elected as Senator at the Society of Women Engineers - a not for profit organization with over 42,000 global members and the world's largest advocate and catalyst for change for women in engineering and technology. She is also a member of the Society for Industrial and Organizational Psychology. Learn more about her work and get in touch at www.ThatStatsGirl.com.

\section{Dr. Michelle Soledad, The Ohio State University}

Michelle Soledad is a Lecturer in the Department of Engineering Education at The Ohio State University. She holds degrees in Electrical Engineering (BS, ME) from the Ateneo de Davao University (ADDU) in Davao City, Philippines, and in Engineering Education $(\mathrm{PhD})$ from Virginia Tech. Her research interests include learning experiences in fundamental engineering courses and data-informed reflective practice. Michelle's professional experience includes roles in industry and academia, having worked as a software engineer, project lead and manager before becoming Assistant Professor and Department Chair for Electrical Engineering at the Ateneo de Davao University.

\section{Dr. Tamoghna Roy, DeepSig Inc.}

Tamoghna Roy works as a Principal Engineer at DeepSig where he is responsible for creating novel machine learning solutions to classical wireless communication problems, thus enabling the next generation of wireless systems. Tamoghna received his PhD and MS in Electrical Engineering from Virginia Tech in 2017 and 2014 respectively.

\section{Dr. Homero Murzi, Virginia Polytechnic Institute and State University}

Homero Murzi is an Assistant Professor in the Department of Engineering Education at Virginia Tech with honorary appointments at the University of Queensland (Australia) and University of Los Andes (Venezuela). He holds degrees in Industrial Engineering (BS, MS), Master of Business Administration (MBA) and Engineering Education (PhD). Homero is the leader of the Engineering Competencies, Learning, and Inclusive Practices for Success (ECLIPS) Lab. His research focuses on contemporary and inclusive pedagogical practices, emotions in engineering, competency development, and understanding the experiences of Latinx and Native Americans in engineering from an asset-based perspective. Homero has been recognized as a Diggs Teaching Scholar, a Graduate Academy for Teaching Excellence Fellow, a Global Perspectives Fellow, a Diversity Scholar, a Fulbright Scholar, and was inducted in the Bouchet Honor Society.

\section{Prof. Tamara Knott, Virginia Polytechnic Institute and State University}

Tamara Knott is Associate Professor of Engineering Education at Virginia Tech. She primarily teaches Engineering Foundations classes to first year engineering students. Her interests include assessment and pedagogy. Within ASEE, she is a member of the First-year Programs Division, the Women in Engineering Division, the Educational Research and Methods Division, and the Design in Engineering Education Division. She is also a member of the Society of Women Engineers (SWE) and is the Faculty Adviser for SWE at VT. 


\title{
A semester like no other: Use of Natural Language Processing to Summarize Sentiments from End-of-Semester Surveys on the Experience of Changing Learning Environments due to COVID-19
}

\author{
Abstract \\ In response to campus closures due to COVID-19, the learning environment in a foundational engineering \\ course unexpectedly shifted from hands-on, collaborative work to remote delivery, accomplished within a \\ short period of time. Through end-of-semester course surveys, students were asked open-ended \\ questions to get feedback about their experience with the goal of using student feedback for curriculum \\ planning and improvement should there be continued need to facilitate the course remotely in subsequent \\ semesters. However, with 1,170 responses, the volume of data made it challenging to analyze, interpret \\ and use the feedback for decision-making for following semesters. To address this challenge, we utilized \\ Natural Language Processing (NLP) based techniques - algorithmic ways to analyze, interpret, and \\ present words and sentiments from student responses visually, to inform a novice-led analysis to \\ ultimately help with course planning for future semesters.
}

Keywords: COVID-19, First-Year Engineering, Machine Learning, Sentiment Analysis, Assessment

\section{Introduction}

We consider integrating student feedback and experiences into course planning as critical, since students are an important stakeholder in the learning environment (Lattuca and Stark 2009). Our approach of using heuristic approaches through automated tools to enable faster preliminary insights from student responses may be a first step towards helping instructors and administrators make informed decisions for their courses. Amplifying students' voices and allowing them to significantly contribute to course improvement has been demonstrated to increase engagement and promote deeper conceptual understanding and learning (Murzi, 2019). However, even though end-of-semester surveys are a rich, readily-available source of student experiences, they may often be missed opportunities due to the time and resources required to analyze them. This is especially true in large foundations of engineering classrooms, where enrollment numbers can be in the 100s. Analyzing open-ended responses from such classrooms, manually, would require not only time, but also training and person-hours from experienced qualitative researchers.

Indeed, Creswell (2014) describes how qualitative data analysis involving hand-coding is often a "laborious and time-consuming process even for data from a few individuals" (pg. 195). Teddlie and Tashakkori (2012) had previously noted that for larger sample sizes and more complex analyses computers may be more essential. Methods experts have thus long recommended the use of computers 
to aid analysis. For example, Creswell (2014) and Guest (2012) both described the utility of computer based qualitative data analysis to organize, sort, and search for information in the text thus helping researchers to access the entire text more conveniently. Advancements in data processing techniques, especially with the advent of tools such as machine learning, and other algorithmic means to understand large datasets can lend themselves as plausible solutions to the "big-data" problem within engineering education. Natural Language Processing techniques are one such computer-based, albeit heuristic, approach which can be used as exploratory tools to analyze qualitative datasets, thereby helping researchers make meaning out of large volumes of interview transcripts.

Recognizing the limitations of heuristic models, and that such modeling of natural languages may not yield insights even close to nearly as deep as qualitative researchers may yield, we propose an algorithminformed, novice-led approach to analyzing large qualitative datasets which can inform deep dives into datasets. The role of novice researchers as important in engineering education was demonstrated through a paired novice-led thematic analysis approach adopted by Montfort, et. al. (2013). Describing the approach, Montfort, et. al. (2013) claimed that researchers working together to analyze qualitative datasets provide a richer and more rigorous analysis which is theoretically sound when the analysis is led or guided by a relative novice in the content area. This approach was expanded upon in work by Bhaduri (2018) where they demonstrated the use of Natural Language Processing in facilitating exploratory analysis of a large interview dataset by a novice researcher, to then inform a larger qualitative research group.

\section{Purpose}

The purpose of this paper reporting on our work-in-progress research is to elaborate on our approach to learn from a Natural Language Processing-based, novice-led approach to understanding preliminary insights from open-ended responses on changes to a foundational engineering course due to disruptions as a result of the covid-19 pandemic. The study was conducted in a predominantly white, large public land grant university in the Southeast United States.

\section{Impact of the pandemic on a Course on Fundamentals of Engineering}

Fundamentals of Engineering is a two-course sequence for first-year general engineering students at a large public land grant university in the Southeast United States. The spring semester sequence takes a hands-on approach to facilitate the learning of engineering design, mathematical modeling, contemporary software tools, and professional practices and expectations. In response to campus closures due to COVID-19, the learning environment unexpectedly shifted from hands-on, collaborative work to remote delivery, accomplished within a short period of time. Through end-of-semester course surveys, students were asked open-ended questions to get feedback about their experience with the goal of using student 
feedback for curriculum planning and improvement should there be continued need to facilitate the course remotely in subsequent semesters.

When the learning environment shifted to remote delivery after spring break, due to the onset of the pandemic in 2020, student teams had only completed the first round of physical prototypes and were poised to work with their teams to begin improvements on their designs. Depending on the project, some teams had completed their mathematical modeling while others had not yet begun. While all teams used basic sketching, the amount of CAD modeling that had been done varied. The major challenges faced by instructors as the medium turned unexpectedly virtual included: (1) enabling teams to continue to work together given varying locations; (2) internet connectivity and lack of experience with appropriate software tools; (3) the inability to physically work with peers on the second prototype; (4) the lack of access to tools and materials for prototyping; and (5) needing to learn and use modeling software remotely. Considering all these challenges, the instructors collegially decided to enable asynchronous delivery of learning experiences, supplemented by virtual office hours.

To help students transition into the virtual mode of teamwork while experiencing the pandemic, instructors provided additional learning resources, including videos and narrated presentations, that students could access at their preferred time and pace supplemented within the course's learning management system. Some assignments were modified; for example, a team project that entailed building a drone prototype was redesigned into developing a brochure that showcased the characteristics of the drone designed by students. Despite changes to hands-on assignments and an inability to physically engage with peers, some students elected to build and test their prototypes on their own, with guidance from their instructor. Some instructors also integrated reflection assignments, with the goal of providing an outlet for students to process the learning circumstances they unexpectedly found themselves in due to the pandemic. Examples are shown in Figure 1. 

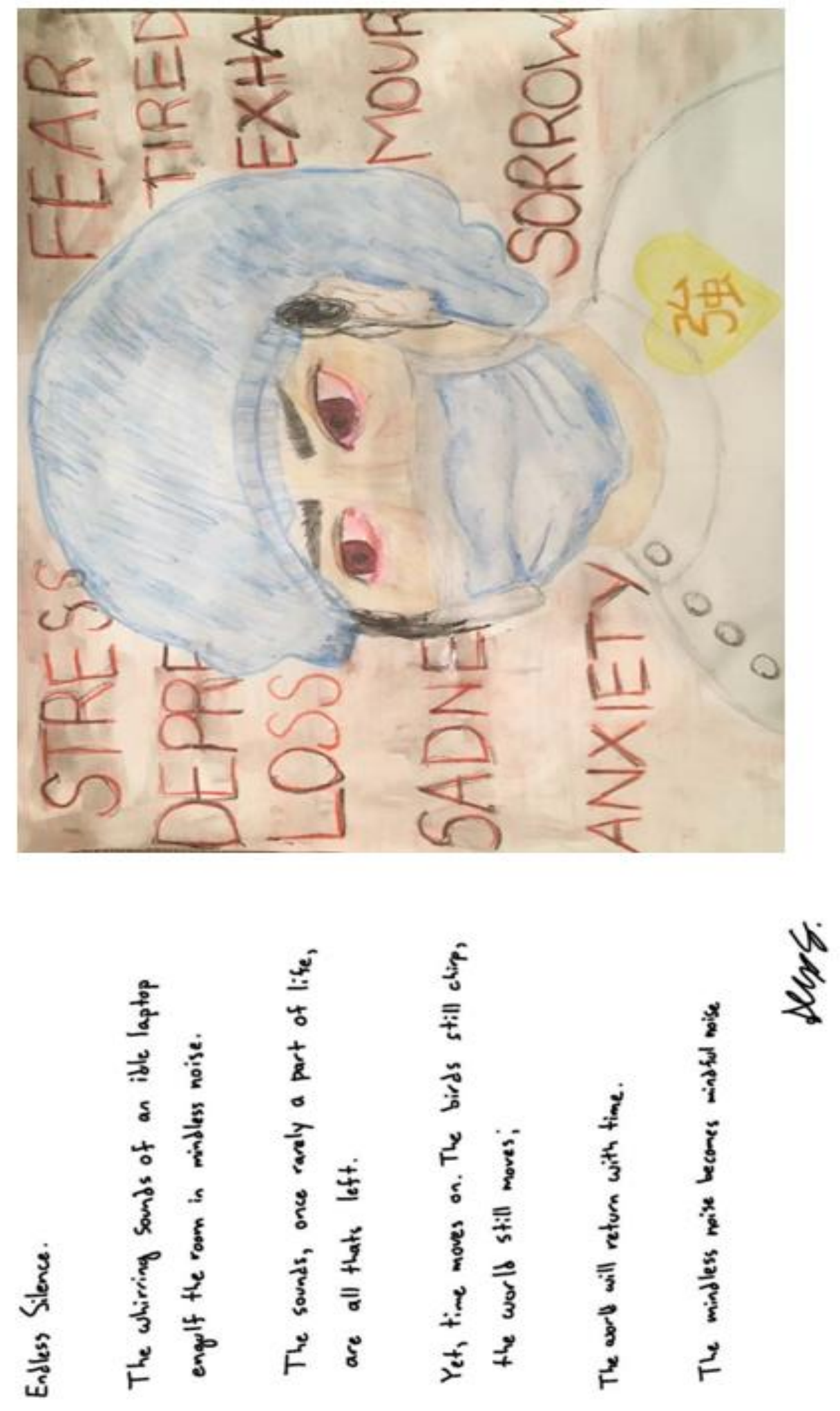

Figure 1. Examples of creative assignment submissions - reflections on moving online due to COVID-19 
At the end of the course, students were asked to describe their learning experiences in light of the transition from in-person to online. However, with 1,170 responses, the volume of data made it exceptionally challenging to analyze, interpret and use the feedback for decision-making. Based on the creative assignment submissions during the course, and especially given the ongoing pandemic, the researchers on this project felt it important to find ways to make meaning of the open-ended sections of the course evaluations, and use them to drive curricular redesign for coming semesters. However, limitations of time and the largely exploratory nature of the dataset seemed intimidating from a resource perspective to take a deep-dive qualitative analysis without any starting points. To address this challenge, we utilized Natural Language Processing (NLP) techniques as a novice-led approach to analyze, interpret, and present data constructively for use as preliminary input into further qualitative deep-dives which would all ultimately inform course planning for coming semesters.

\section{Natural Language Processing augmented Novice-led Approach}

A novice is a person who "has no experience with the situations in which they are expected to perform tasks" (Benner, 1982). The novice is thus at the basic proficiency level for skill acquisition, with limited information and prior experience related to a task at hand (Montfort, et. al. 2013). For large qualitative datasets in engineering education where little is known, such as in the case of students' experience with virtual classrooms through an ongoing pandemic, a novice-led approach to analyzing data may seem a good fit. In the case of our research, we augmented the novice-led analysis with the use of Natural Language Processing. Natural Language Processing has previously been used successfully to make meaning of qualitative datasets in Engineering Education contexts (e.g., Bhaduri and Roy 2017; Andersson, Dryden, and Variawa 2018).

As elaborated upon in the previous section, Natural Language Processing techniques may be used to facilitate the analysis and interpretation of large volumes of qualitative data (Soledad et al. 2017; Bhaduri, 2018), allowing analysis of 1,170 student responses. We used NLP techniques to process responses to open-ended questions regarding this modified approach to the course. Our approach had two main steps, as illustrated in the diagrammatic overview of our methods in Figure 2 below. As can be seen in the figure, we first determined words or phrases that appeared most frequently in responses to gauge what students referred to the most. Once we isolated these words of interest, we used them to identify sentiments conveyed through responses which included those words. 


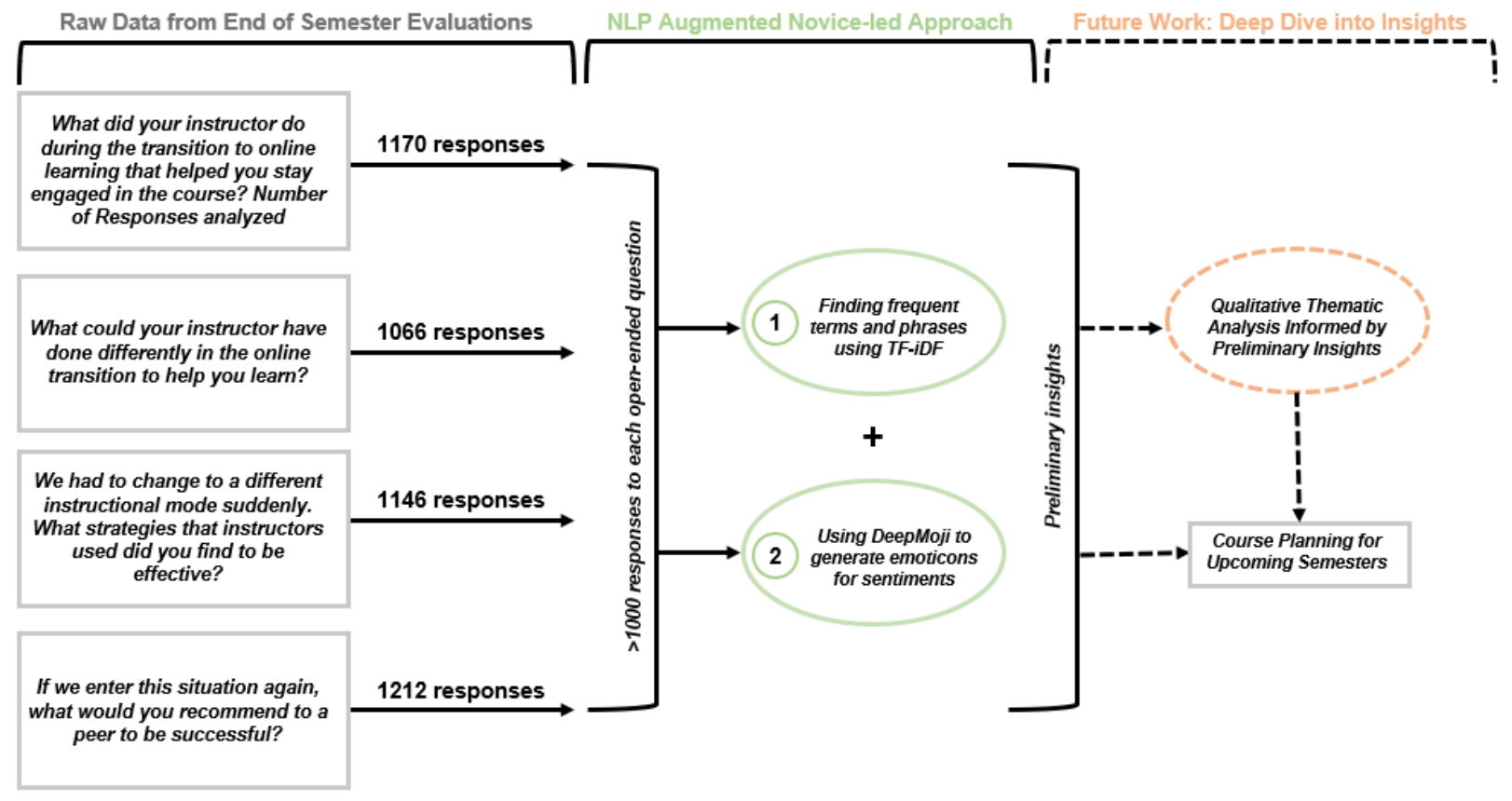

Figure 2. Diagrammatic overview of the steps in this research

\section{Finding Frequent Terms and Phrases using TF-iDF}

To isolate these frequently-used words across the excerpts, we conducted a term frequency-inverse document frequency-based (tf-idf) analysis. Tf-idf is a popular method that goes beyond the word frequency to not only compute frequency of words in a particular document, but multiplies this frequency count with the inverse document frequency (i.e., frequency of the word per response), which leads to lower tf-idf scores for oft-repeated words such as articles ( $a$, an, the) which occur in large instances in both single documents, and in the entire corpora (Shi, Xu, and Yang 2009; Variawa, McCahan, and Chignell 2013). Looking at the responses to the following open-ended questions, we identified words with highest tf-idf scores, shown in Table 1.

\section{Table 1. Words with highest tf-idf scores for each question}




\begin{tabular}{|c|c|c|}
\hline $\begin{array}{l}\text { Top } 5 \text { terms } \\
\text { using tf-idf } \\
\text { scores } \\
\text { (per question) }\end{array}$ & $\begin{array}{l}\text { Percentage of } \\
\text { Responses } \\
\text { with Term }\end{array}$ & Example of response \\
\hline \multicolumn{3}{|c|}{$\begin{array}{l}\text { What did your instructor do during the transition to online learning that helped you stay engaged in the course? Number } \\
\text { of Responses analyzed: } 1170\end{array}$} \\
\hline assignment & $30 \%$ & \multirow{5}{*}{$\begin{array}{l}\text { "I enjoyed the videos <Instructor> sent to his class explaining the goals } \\
\text { of each week following the transition online. The videos he provided } \\
\text { made each assignment clear, and I had no problem following them." }\end{array}$} \\
\hline video & $28.72 \%$ & \\
\hline weekly & $22.05 \%$ & \\
\hline post & $18.03 \%$ & \\
\hline update & $11.03 \%$ & \\
\hline \multicolumn{3}{|c|}{$\begin{array}{l}\text { What could your instructor have done differently in the online transition to help you learn? } \\
\text { Number of Responses analyzed: } 1066\end{array}$} \\
\hline assignment & $21.43 \%$ & \multirow[t]{5}{*}{$\begin{array}{l}\text { "I thought it was a good transition to online, and the assignments } \\
\text { weren't overbearing but still gave me an understanding of what } \\
\text { engineers might do" }\end{array}$} \\
\hline team & $9.2 \%$ & \\
\hline transition & $9.93 \%$ & \\
\hline great & $5.21 \%$ & \\
\hline class & $9.93 \%$ & \\
\hline \multicolumn{3}{|c|}{$\begin{array}{l}\text { Question: We had to change to a different instructional mode suddenly. What strategies that instructors used did you } \\
\text { find to be effective? Number of Responses analyzed: } 1146\end{array}$} \\
\hline lecture & $26.37 \%$ & \multirow{4}{*}{$\begin{array}{l}\text { "I thought it was great how professors still tried to maintain a } \\
\text { classroom atmosphere with zoom meetings and how they would } \\
\text { record them in case you couldn't make it to class" }\end{array}$} \\
\hline video & $23.45 \%$ & \\
\hline assignment & $16.02 \%$ & \\
\hline class & $16.11 \%$ & \\
\hline
\end{tabular}




\begin{tabular}{|c|c|c|}
\hline student & $13.98 \%$ & \\
\hline \multicolumn{3}{|c|}{$\begin{array}{l}\text { Question: If we enter this situation again, what would you recommend to a peer to be successful? } \\
\text { Number of Responses analyzed: } 1212\end{array}$} \\
\hline work & $33.31 \%$ & \multirow{5}{*}{$\begin{array}{l}\text { "I would recommend staying very organized. Being online is good for } \\
\text { students who can keep track of their work easily. There is no one } \\
\text { reminding you what assignments are due or what topics you have to } \\
\text { cover that week." }\end{array}$} \\
\hline stay & $24.42 \%$ & \\
\hline schedule & $18.19 \%$ & \\
\hline assignment & $20.43 \%$ & \\
\hline time & $17.52 \%$ & \\
\hline
\end{tabular}

Consider the prompt, "What did your instructor do during the transition to online learning that helped you stay engaged in the course?" As seen from Table 1, 30\% of the responses used the word 'assignment' to describe what helped them stay engaged in the course. The word 'assignment' and its variants were isolated as a word of interest for this question. However, tf-idf was unable to go beyond capturing the frequency of use of a word. For example, the word 'video' was used in both responses - "All the instructions... and reasoning for doing the assignments were pretty clear with both the videos and templates" and "The assignments just seemed like busy work." While the latter of these two responses presents a critique of the course, the other praises the instructor for their clarity in communication.

\section{Using DeepMoji to generate emoticons for sentiments}

Since tf-idf only involves word frequency analysis, we followed up tf-idf with an approach for sentiment analysis to further understand the context of the responses where frequently-used words appeared. For this, we extended the use of the DeepMoji algorithm which essentially allows text to be represented as emojis. Thus, for our next step, we wanted to understand sentiments associated with a particular word of interest. DeepMoji (Felbo et al. 2017), a deep learning-based tool, was programmed to assign emojis to responses containing words of interest. For example, for the word, 'video', the top 5 emojis assigned

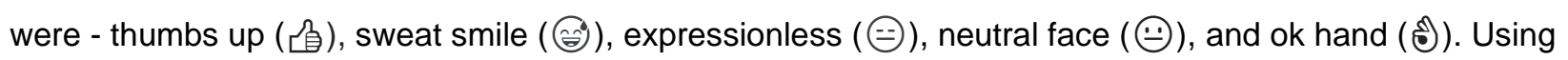
DeepMoji, the algorithm assigned a thumbs up emoji with a 30\% confidence to the student response, "I enjoyed the videos < Professor> sent to his class explaining the goals of each week following the transition online. The videos... made each assignment clear." This allowed us to gauge the range of sentiments conveyed through the responses, and which strategies did or didn't work based on students' responses. 
A combination of insights from the frequency of oft-used phrases and the sentiments associated with them can be used by qualitative researchers to do a deep dive into understanding the students' experiences. For example, isolating all responses with the word assignment, and going through only those comments assigned a neutral face emoji or the ok hand emoji would give researchers (or instructors) insight into comments, feedback and critique related to assignments in the course. Similarly, other words and phrases can be isolated and understood. As is expected, this technique does not attempt to replace the qualitative researcher, but rather help them augment their exploratory research, especially for large datasets by providing information about starting points through preliminary insights. Through the use of Natural Language Processing, we were thus able to begin to uncover important preliminary insights about students' perspectives on the transition to remote learning.

\section{Future Work}

As our first-year class sizes get larger, so do datasets comprising student feedback collected in those classes. Time- and resource-effective methods are urgently needed, especially as we leverage student experiences as input into course redesign and planning. Such efforts were reinvigorated given the ongoing pandemic, and is likely a trend that will continue to leverage upon this previously-missed opportunity of using responses to open-ended items in student evaluations to incorporate student feedback into course planning as we move beyond the pandemic. Natural Language Processing is a set of heuristic computational linguistic techniques to make meaning from large qualitative datasets using algorithmic insights. However, as with any automated, algorithmic approach to understanding humans, the limitations of these techniques must be acknowledged. One common limitation is related to semantics or context of languages. Jurafsky and Martin (2007) described semantics as the knowledge of meaning. Jurafsky and Martin further noted that language processing systems differ from other data processing systems since they use knowledge of the language. Training an algorithm to understand knowledge, contexts, and meaning is a non-trivial task, and the insights obtained should not be blindly used to drive decision-making. Such blind/black-box approaches may exacerbate ethical considerations of datause that are already heavily under scrutiny, and rightly so (e.g., Algorithms of Oppression by Safiya Noble looks into how algorithms can indeed perpetuate stereotypes and racism). The contribution of this work is thus in recommending a novel approach, but also cautioning engineering educators and emphasizing on the need to follow-up on insights yielded by heuristic algorithmic approaches with qualitative research. Thus, we emphasize upon extending the novel uses of Natural Language Processing to augmenting novice-led exploratory thematic analysis of engineering education data. We hope that this work is of value to engineering education researchers and instructors alike, who may find value in such approaches to tackle large qualitative datasets, such as in making meaning from open-ended responses submitted end-ofsemester by students in a large foundational engineering course during times of unprecedented pandemic and change, truly a semester like no other! 


\section{References}

Andersson, Eric, Christopher Dryden, and Chirag Variawa. 2018. "Methods of Applying Machine Learning

to Student Feedback through Clustering and Sentiment Analysis." In Proceedings of the Canadian

Engineering Education Association (CEEA) Conference. Vancouver, BC.

Bhaduri, S. (2018). NLP in Engineering Education-Demonstrating the use of Natural Language

Processing Techniques for Use in Engineering Education Classrooms and Research (Doctoral dissertation, Virginia Tech).

Bhaduri, Sreyoshi, and Tamoghna Roy. 2017. "A Word-Space Visualization Approach to Study College of Engineering Mission Statements." In 2017 IEEE Frontiers in Education Conference. Indianapolis, IN: IEEE.

Creswell, John W. 2014. Research Design: Qualitative, Quantitative, and Mixed Methods Approaches. 4th ed. Thousand Oaks: SAGE Publications.

Felbo, Bjarke, Alan Mislove, Anders Søgaard, lyad Rahwan, and Sune Lehmann. 2017. "Using Millions of Emoji Occurrences to Learn Any-Domain Representations for Detecting Sentiment, Emotion and Sarcasm." In Proceedings of the 2017 Conference on Empirical Methods in Natural Language Processing. Copenhagen, Denmark. https://doi.org/10.18653/v1/D17-1169.

Lattuca, Lisa R., and Joan S. Stark. 2009. Shaping the College Curriculum: Academic Plans in Context. 2nd ed. San Francisco, CA: Jossey-Bass.

Montfort, Devlin B., Geoffrey L. Herman, Shane A. Brown, Holly M. Matusovich, and Ruth A. Streveler. 2013. "Novice-Led Paired Thematic Analysis: A Method for Conceptual Change in Engineering." In 120th ASEE Annual Conference \& Exposition, 23:1. Atlanta, GA: American Society for Engineering Education. Murzi, Homero G. 2019. “Using Continuous Feedback as an Alternative Form of Students' Evaluation of Teaching - UQ ESpace." In . Cape Town, South Africa: Research in Engineering Education Network. https://espace.library.uq.edu.au/view/UQ:8aa40c2.

Shi, Congying, Chaojun Xu, and Xiaojiang Yang. 2009. "Study of TFIDF Algorithm." Journal of Computer Applications 29 (6): 167-70.

Soledad, Michelle, Jacob Grohs, Sreyoshi Bhaduri, Jaime Williams, Jennifer Doggett, and Steven Culver. 2017. "Leveraging Institutional Data to Understand Student Perceptions of Teaching in Large Engineering 
Classes." In Frontiers in Education Conference (FIE), 2017. Indianapolis, IN: IEEE.

Teddlie, Charles, and Abbas Tashakkori. 2012. "Common 'Core' Characteristics of Mixed Methods

Research: A Review of Critical Issues and Call for Greater Convergence." American Behavioral Scientist 56 (6): 774-88. https://doi.org/10.1177/0002764211433795.

Variawa, C, S McCahan, and M Chignell. 2013. "An Automated Approach for Finding Course-Specific Vocabulary." In . Atlanta, GA: American Society for Engineering Education. 